\title{
Rolf Knieper Zurichtung nationaler Politik durch internationales Recht?
}

\author{
DIE BEREITSCHAFTSKREDITABKOMMEN DES INTERNATIONALEN \\ WAHRUNGSFONDS ${ }^{*}$
}

\section{Einleitung}

Unter den Organisationen der Vereinten Nationen wächst dem Internationalen Währungsfonds in Washington D. C. zunehmend eine Sonderstellung zu: er beeinflußt und steuert in zunächst einzelvertraglichen und zunehmend Rechtsnormenstandard gewinnenden Formen die Wirtschaftspolitiken der Mitgliedsnationen. Der Einfluß greift direkt: der Respelst vor nationalscaatlicher Souveränität beschränkt sich auf die Beachrung diplomatischer Verkehrsformen, inhaltlich unterwerfen sich die Partnerscaacen einheitlichen, politischen Bedingungen; diese sind aus einer homogenen Wirtschaftspbilosophie entwickelt, die ihre wesentlichen Impulse vom Chicago-Liberalismus und -Monetarismus empfängt und sich im Gewande unpolicisch-technokratischer Sachkomperenz präsentiert. Mit diesem akzeptierten Selbstverständnis setze der IWF seine Bedingungen in Südafrika wie in Tansania, in Brasilien wie in Peru, in der Türkei wie in Israel, in Indonesien wie in Pakistan. Dem Begreifen der Welt als Markt, auf dem abstrakr, gleichförmig. Waren gerauscht werden und sonst gar nichts, entspricht das abstrahierende Ignorieren einer NordSüd-Problematik, so daß Ghana und Frankreich, Porrugal, Iralien und Großbritanrien mit eben denselben Bedingungen überzogen werden. Auch der Volksrepublik China, die sich in Moment um Beitritt zum IWF bemüht, werden keine neuartigen wirtscbaftspolitischen Bedingungen auferlegt werden.

Wie immer täuscht das Bild vom unpolitischen Charakter der stechnokratischen Rarionalitäı. Bereits die Länderbehandlung folgt nichr dem glatten Prinzip der Blindheit gegenüber der innenpolitischen Situation: Militärdiktaturen wie Süd-Korea oder Brasilien gelen als vorbildlich, die Kooperation mit Chile ist erst seit dem Sturz der Volksfront ganz zufriedenstellend, noch im Mai 1979 erhielt Somozas Nicaragua einen Kredic, um odas Wirtschaftsprogramm der Regierung zu unterstüzzen "', die Vereinigten Staacen passen sich Bedingungen nicht an, die erwa in Großbritannien für selbstverständlich zumurbar gehalren werden. Auch der Inhalt der IWF-Richtlinien kann wirtschaftspolicische Parteilichkeit nicht verleugnen: Erhöhung des Steuerauflommens, Abbau der Scaatsverschuldung, Veneuerung von Sozialleistungen bei ihrer gleichzeirigen Verringerung, konkrecer: die Aufhebung - wo in sozialreformerischer Absicht durchgesetzt - freier medizinischer Versorgung, einer Preisbindung für Grundnahrungsmittel Brot oder Reis, eines Schulmilchprogramms für arme Kinder bildeo feste Programmpunkre eben dieser monetaristisch-liberalen IWF-Philosophie, die allerdings in ihrer konkreten Brutalität nicht fixier werden und auch nicht fixiert werden müssen: die zwangsläufigen

\footnotetext{
"Für Günther Busch zum 50. Geburssag.

Material zu dicsem Aufsatz, habe ich wahrend eines Forsichungssemesters gesammele das ich dank der finanzicllen Unterstüzung durch die Liniversizäi Bremen in Washington D. C./USA verbringen konnte. Für krivische Diskussionen danke ich besonders U. Mückenberger. I IMF Survey May 21, 1979, 5. 1 2 .
} 
Ausfühnungen von abstrakten IWF-Programmen bleibc den nacionalen Regierungen vorbehalcen. Wie umfassend policisch die IWF-Programme aber sind, belegen nationale Unruhen, die sich häufig nach dem Abflug einer IWF-Mission entwickeln - Peru, Portugal, die Türkei sind jüngere Beispiele - und die nicho selten zu Regierungsumstürzen führen. Nicht ohne einen gewissen Beweger-Stolz berichtete mir ein Mitarbeiter des IWF, daß die Errichtung der Militärdikcatur in Ghana unmictelbare Folge einer IWF-Mission gewesen sei: eine demokratisch-parlamentarische Regierung sei nicht in der Lage gewesen, die Rigorosität der geforderten Maßnahmen durchzusetzen.

Diese Entwicklung kontrastier eigenümlich zu dem Zusammenbruch der ursprüngliçen Konzeption eines Internationalen Währungsfonds, bie - nach den Erfahrungen mir der Wirschałts- und Währungskrise der joer Jahre - darauf angelegt war, Währungspolitik aus dem narionalstaarlichen Konkurrenzkampf zu lösen und durch den Aufbau einer Weltwährungsbehörde zu internationalisieren: Mit der 2. Novellierung der WWF-Statuten, die 1978 in Kraft getreten ist ${ }^{12}$, wurde diese Konzepcion auch juristisch zerstört und Währungspolitik re-nationalisiert - eine Situation, die dem inzwischen erreichten Grad der Welcmarkiverflechrung kaum entspricht:

Das bestehende Vakuum füllt weirgehend ein privater Kapitalmarkr aus, der von international agierenden Banken kontrolliert wird. Diese entgehen ihrerseits jeder staatlichen oder internationalen Kontrolle und stellen auf diese Weise lediglich ein prekäres, ständig durch Konkurrenz bedrohtes Gleichgewicht her, das bereits durch minimale Krisen - wie erwa den Herscatt-Zusammenbruch - weltweir aus den Fugen zu geraten drohc. Regionale Ersatzkonstruktionen wie die 1977 in Kraft getretene Europäische Währungsunion (EWU) versuchen, auf die Labilicät der Situacion zu reagieren, ohne sie ausräumen zu können ${ }^{2}$ und auch die Beistandskredirabkommen des IWF verdanken sich der zunehmenden Integration des Weltmarkes. Sie stellen quasi unvollkommene Äquivalente zur ursprünglichen Konzeption einer internationalen Währungspolitik dar. Der IWF konzentriert seine Mitrel und Kapazität weitgehend darauf, jeweils nationale Austeritätsprogramme durchzusetzen oder doch zu unterstützen. Damic stellt sich in gewisser Weise eine internacionale Harmonisierung von Wirtschaftspolitiken her. Die in Art. I der IWF-Sranten genannten Ziele - ausgeglichenes Wachstum des Welthandeis, hoher Beschäfrigungsgrad, steigende Massen-Realeinkommen, Encwicklung der Produkcionskraft, Stabilität der Währungen - sind durch Ausceritärspolicik jedoch kaum gefördert worden. Vielmehr sind Massen-Arbeitslosigkeic, Verelendung und Inflacion häufige Folgen von Austeritässpolitik, wobei bisher jeder Nachweis dafür fehlt, daß solche negativen Folgen lediglich als Preis für einen langfriszigen Wirrschaftsaufschwung zu encrichten seien: keines der unter IWF-Programmen operierenden Länder läßt sich als Beispiel dafür zitieren, daß nach einer Phase von »Reinigungs $*$-Ausrerität die Beschäfrigung und das Massen-Realeinkommen auf der Grundlage des Programms gestiegen sei'. Im IWF selbst sind diese Konsequenzen nicht unbekannt: sie

12 Die englischen und deutschon Fassungen des IWTF-Obereinkommens sind abgeớruckt in BGBI. $\mathrm{Il}$ ( 1952 ). S. 637 (Erstfassung) und BGBl. II (1978) S. 13 ff. (F2ssung nach der 2. Novellierung, die am 1. 4. 1978 in Kraft ge(reten ist).

2 Vgl. dazu ausfübrlich mcine Arbcitcr: Multinationales Unternehmen und Nationalsuat im Kontliks? - Am Bcispiel des Internationalen Währungssystems. In: Däubler/Wohlmurb (Hrsg.), Transaationalc Konzerme und Weltwinschaftsordaung. 1978, S. 80 ff; Der Währungsfonds strotzt vor Impotenz, in: Fraokfuner Rundschau vom 22. 1. 1978.

$3 \mathrm{~V}_{\mathrm{g}}$. erwa die 'keynesianisch, argumenierende Fallstudic von E. Eshag und R. Thorp, Economic and Social Consequerices of Orthadox Economic Policies in Argentina in the Post-War Years, in: Bullecin of the Oxlord Universiry Instiute of Economics and Statistics, Vol. 27, No. I, Febr. 1965, S. I ff; 
werden insgesamt - also unter Einschluß der Inflation - als norwendig betrachret, was die Anfangsphase angeht, während die auch langfristig ausbleibenden Verbesserungen der wircschaftlichen Siruation mic dem eher lapidaren Hinweis erklärt werden, daß die Programme auf kurze bis mitclere Frist angelegt seien und bisher noch kein Land den Arem für eine langfristige Fortührung gehabr habet.

Das Mitrel, dessen sich der IWF zur Durchsetzung seiner wirtschaftspolitischen Vorstellungen in Mitgliedsländern bedient, werden stand-by-arrangementse (Bejstandskredirabkommen) genannt: hinter diesem Begriff verbirgt sich - untechnisch gesprochen' - die Einräumung einer Kredit-Linie, die ausgenutzr werden kann, sofern das Land bestimmte Bedingungen einhält, welche in einem gesonderten Dokument, dem letter of intent (Absichtserklärung) festgelegt sind. Die Erklärung ist in die Form eines Briefes gekleidec, welcher lediglich vom Finanzminister oder Zencralbankpräsidencen des kreditnehmenden Landes uncerschrieben wird, obwohl er die ausgehandelten Bedingungen enthält.

Der folgende Beitrag dient der Darstellung des regelmäßigen Inhalts (II) und der juriscischen Qualifizierung (III) solcher Abkommen sowie einer anschließenden Kritik, die juristische, politische und ökonomische Elemente vereint (IV). Die Präsentarion erfolgt auf der Grundlage von Modell-Abkommen und -absichtserklärungen, wie sie in der Arbeit von J. Gold enrwickelt worden sind ${ }^{6}$, einer Sammlung von Abkommen, die über einen Zeitraum von erwa zehn Jahren abgeschlossen worden sind, und zwar mit so verschieden strukturierten Ländero wie Großbritannieo, Neu-Seeland, Argentinien, Peru und Italien, sowie endlich einer Reihe von Diskussionen, die icb mit Angestellten des TWF geführt habe. Nach meinen bisherigen Erkenntnissen reichr eine solche Materialsammlung zur Analyse aus: Obwohl ein Angestellter des IWE ausdrücklich davor gewarnt hat, die Beistandskreditabkommen unter den Gesichtspunkten von Uniformisierung und Generalisierung zu analysieren, da auf diese Weise spezifische Konscellationen außer ache bleiben müßten, wie ctwa die exakre Problemdiagnose des Defizits des öffenclichen Sektors (die Verschuldung der Sozialversicherung oder der öffentlichen Unternehmen oder des ,Infrastrukrurbereichs s), wie etwa die Etabliertheir der Beziehungen des Micgliedes zum IWF, wie erwa die Tranchenteile, innerbalb derer das Stand-byArrangement verbandelt werde, oder wie etwa die Besonderheit eines Defizit-Landes im Hinblick auf seine fakcische Währungskonverribilizät, trozz dieser Warnung - so versichert ein anderer Angestellter - hat der IWF, wo immer er hinkommh das gleiche Rezepr für die Probleme eines Landes anzubieten. Tarsächlich förderr eine Synopse der mir zugänglichen Absichtserklärungen den Eindruck der Uniformität bei außerordentlicher Unterschiedenheir der beteiligten Länder. In jedem uncersuchten Abkommen steht im Vordergrund, die Komplexië̈ der wirtschaftlichen Probleme eines Landes zum Zwecke der Uniformierbarkeit zu reduzieren auf ein Zahlungsbilanzungleichgewicht, wobei es schon als Konzession erscheint, verschiedene Begründungen für diese Situacion zuzulassen - also etwa Rückschritte in der Produktivitätsentwicklung, soziale Unruhen, die Verschlechrerung der terms of

ebenfalls: T. Hayzer, Aid as Imperialism, 1970; C. Payer, The Debr Trap: The Internarional Monetary Fund and the Third World, 1974. G. Leithäuser. Der Internacionale Wahrungsfonds (WWF) und die eingeschränke Souveräricät narionalstaalicher Wircschafespolisik in seinem Einflußbercich, in: Bläıer fïr deutsche und internacionale Politik, $/ 1978, \mathrm{~S} .290 \mathrm{H}$.

4 Als schriftiche AuBcrung in diesen Zusammenhang: A. S. Gerakis, Recession in the Inirial Phase of a Srabilization Progrnm: The Experience of Finland, IMTF Staff Papers Vol. XI, No. 1, Nov. 1964, S. 434.

\& Technisch gewähn der WW keinc Kredite, sondem die Bcnutzbarkeit der Fonds-Mirtel komme dadurch zustandc, daß ein Land mil der eigenen Währung fremde Wahrungen kaufı und als ,Rückzahlung die eigene Wiahhrung später wieder kaurt.

6 The Stand-by Arrangements of the International Monetary Fund, 1970. 
trade oder unwetterbedingte Mißernten. Der Schlichtheit der Problemdiagnose entspriche die Zielbestimmung: es geht darum, die Zahlungsbilanzsinuation zu verbessern und das Mitrel, dieses Ziel zu erreichen, soll in einer Verlagerung von inländischer - öffenclicher wie privater - Nachfrage auf Exporbemühungen liegen. Damit isc zum Programm erhoben, was am freien Welthandel kritisiert wird: daß nämlich das Bestreben nach günscigen Handelsbilanzen diesem Handel sein Gepräge gebe und nicht - wie es in der Rechtfertigungstheorie heißt - die Ausnuzzung relaciver Produkcionsvorteile?

\section{Der Inhalt von Beistandskreditabkommen}

Den ersten offiziellen, zu einem Beistandskreditabkommen führenden Schritt unternimmi niche der IWF, sondern das kredit-suchende Mirgliedsland. Zu einem solchen Schritt wird ein Land genörigt, wenn es - aus welchen Gründen aucb immer - auf Importe nichr verzichten kann und keine Möglichkeit hat, die Importe zu finanzieren, wobei die Arr der Finanzierung prinzipiell gleichgültig ist ${ }^{8}$ : Sie kann durch Ansammlung von Währungsreserven erfolgen, die ihre Grundlage im Export hat, in Einkünfren aus Invescitionen im Ausland, die also durch national bestimmte Wirtschaftstätigkeit verdiente sind. Die Währungsreserven können ebenfalls angesammelt sein als Ergebnis von Kapitalimport, soweit dessen Ergebnisse sich durch Gewinntransfer nicht umkehren. Es kann sich ferner um Finanzierung in engerem Sinne handeln, nämlich um Kredite auf dem internationalen Kapicalmarkc, um Staatskredite oder auch um Schenkungen. Endlich kann ein Land seine Importüberschüsse durch Vermehrung der nationalen Währung finanzieren und ist dann auf Währungsreserven weniger angewiesen, wenn nur die narionale Währung aut dem Weltmarkt als Geld akzeptiert wird. Der prominentesce Fall dieser Art der Finanzierung sind die USA, deren Zahlungsbilanz beinabe seit der Encscehung des IWF im Defizic ist, ohne daß in diesem Land bisher Finanzierungsschwierigkeiten entstanden wären.

Erst wenn andere Arten der Importinanzierung scheitern und wenn das Land sich dennoch entschließt, nicht aus dem Weltmark-Zusammenhang auszuscheren in eine Politik nationaler Selbsc-Genügsamkeit, muß es die Einflußnahme auf narionale Wirtschaftspolivik hinnehmen, die mit einem Beistandskreditabkommen notwendig verbunden ist, um sich auf diese Weise eine neue Finanzierungsquelle zu eröffnen. Dabei ist die Höhe dieser Kreditlinie eher gering. Sie schwankt zwischen wenigen Millionen US-Dollar (z. B. 1,5 Mill. $\$$ an Bolivien im Jahre 1959, 25 Mill. $\$$ an Süd-Korea im Jahre 1969 , 75 Mill. $\$$ an Peru im Jahre 1968)9 und knapp 1,s Milliarden Sonderziehungsrechten (z. B. 1,29 Milliarden SZR an Großbritannien im Jahre 1978$)^{10}$, geringen Beträgen also im Verhältnis zum Volumen der internationalen Finanzmärkte, das sicb 1977 auf etwa 700 Milliarden US-Dollar belief"r. Es liegt auf der Hand, daß die Kreditlinie nichr das Problem der Verschuldung eines Landes lösen soll; der Zweck ist vielmehr, Kredicwürdigkeit für den privaren Kapitalmarkc

7 Vgl. nur ecwa J. M. Keynes, Algemeive Thearie dex Beschälúgung, des Zinsen und des Geldes, 1936, S. 3226 .

8 Vgl. Fred L. Block, The Origins of Intcrnational Economic Disorder, 1977, S. $1 \mathrm{ff}, 203 \mathrm{fl}$.

9 Vgl. die austührliche Tabelle bei Gold, S. 20, If.

10 Dies is der höchsre je gezogenc Berag; ygl. Jahresbericht des IWF 1978, S. 92; I SZR enesprach 1978 etwa 1,13 US-S.

1 Im Geschätusberiche der Deutschen Bundesbank werden die Auslandsforderungen der Geschäfubanken der ir höchst enrwickelıen Länder mil 6,0 Milliarden US-S angegcben: Geschäfosbericht fur das Jahr 1977 , S. 19. 
wiederherzustellen. Verschiedentlich wird die Hoffnung ausgesprochen, daß die Gewährung eines Beiscandskredits durch den IWF von den internationalen Geschäftsbanken als Signal für die "Solidicär« narionaler Wirtschaftspolitik aufgefaß̊t werde'

Ein Uberblick über die solche "Solidiät" verbürgende Abkommen läßr zwei Kernbedingungen erkennen, an welche die Gewährung der Kreditlinie geknüpft ist. Zum einen wird die Höchstgrenze der inländischen Kreditausweitung quanrizativ eindeurig festgelegt. Dabei wird regelmäßig unterschieden zwischen der Kreditnachfrage des öffentlichen Sektors einerseits, die teilweise in absoluten Zahlen, teilweise in Steigerungsraten begrenzt wird und die einen zusä̈zlichen restriktiven Einfluß auf die Geldmenge dadurch ausüben soll, daß ihre Befriedigung über den Kapicalmarkı und rich über Zentralbankkredice nachzusuchen zugesichert wird, und dem Kreditangebor des privaten Banksektors an private Haushalte und Uncernehumen andererseits, das ebenfalls quantitativ begrenzt wird, wobei nicht selten bestimmte Sektoren ausdrücklich genannt werden, etwa der privare Wohnungsbau, bestimme wichrige Produkcionsteile oder landwirtschafrliche Sekroren.

Zum zweiten wird die Zusicherung verlangt, den Devisenverkehr zu liberalisieren, d. h. bestehende Restriktionen nicht zu verschärfen, Konverribilizät für langlaufende Zablungen und möglichst auch für den Kapitalverkehr herzustellen, Handelsbeschränkungen nicht zu verschärfen bzw. zu liberalisieren und häufig auch, den Kapitalverkehr zu Liberalisieren's.

Um diese Kernbedingungen ranken sich weitere Bedingungen, die jedoch nicht immer als quancitativ bemessene, harte Durchführungsminima (sog. performance clauses) charakterisiert sind und bisweilen den Charakter allgemeiner politischer AbsichrserkJärungen haben. Diese Unterschiedlichkeir der Ausgestalrung finder sich bei den Zusicherungen über die einzuschlagende Einkommenspolirik wie über die Einnahmepolitik der öffentlichen Hand. Während ecwa in dem rg67er Abkommen mit Neu-Seeland zur Einkommenspolitik jeder Hinweis fehlt, und etwa in dem r 977 er Abkommen mit Argentinien nur eine allgemeine Absichrserklärung abgegeben wird, auf niedrige Lohnabschlüsse hinzuwirken und im öffentlichen Sektor auch durchzuserzen, finder sich erwa im r976er Abkommen mir Großbritannien - im Gegensatz zum r967er, wo ebenfalls nur allgemeine Politikerwägungen angestelit sind - eine ausdrückliche Quanrifizierung, nämlich die Zusicherung, unter dem Sozialkontrakt" Lohnerhöhungen nur im Rahmen von höchsters $10 \% \mathrm{zu}$ tolerieren. Ähnlich diufferierende Angaben lassen sich für die Einnahmepolicik der öffentlichen Hand darstellen: neben allgemeinen Erklänungen, Steuern zu erhöhen und/oder das Eintreibungssystem zu verbessern, finden sich exakte Angaben darüber, welche Steuerarten zu welchem Prozentsatz und mit der Erwartung auf welchen Ertrag erhöht werden sollen, was niche selten - wie etwa im 7 6er Abkommen mir Großbritannien - zusammenfällt mir der Ankündigung, Sieuersenkungen zugunsten der Industrie durchzusezzen, um - so die offizielle Version - Investi-

$12 \mathrm{~J}$. Gold, S. $37 / / 191 / 230$ ebenso F. Braun, Die Zehner-Gruppc - Stellung und Kooperstion der Zehnergruppe im internasionalen Währungssystem, 1970, S. 29 II.

1) Vgl dazu neben dem Muster-Abkommen bei J. Gold, S. 37-64 die folgenden Bereicschafuskreditabkommen: mir Argentinien vom 4. 12. 1958 , ziticn nach Economic Survey, English Edition, Buenos Aircs vom 24. 3. 1959; mit Argentinien vom 23. 8. 1977, unveröffentlicht; mit Großbritannien vom 23. 11 . 1967, ziviert nach: Parliamentary Debates (Hansard), House of Commons vom 30. 11. 1967, Sp. 648; mit Großbritannien vom 15. 12, 1976, Financial Times vom 16. 12. 1976, sowie die unvcröffentlichte Ergänzung vom 14. 12. 1977; mis Italien von 1977, zitierz nach: ll Sols/24 Ore vom 19. 4. 1977; mix Neu-Seeland vom is. 10. 1967, ziticr nach W. Rosenberg, A Guidebook ro New Zealand's Fururc. 1968, 5. 69 If; mit Peru vom 21. 11 1977, Zusammenlassung in: The Andean Repon, December 1977, S. 225 H. 
rionsanreize zu schaffen. Ein weiteres Mittel der Einnahmensteigerung der öffentlichen Hand ist die Gebübrenerhöhung für öffentliche Dienste, wie Elektrizieätsversorgung, öffentlicher Transport etc., und tatsächlich enthalten etwa die Absichtserkärrungen der Regierungen von Neu-Seeland (1967), Argentinien (1958) und Peru ( 977 ) - bisweilen quantifizierte - Erhöhungszusicherungen, die in allen genannten Fällen zusammenfallen mit der Zusicherung, bestimmze Sozialleistungen zurückzunehmen, also z. B. ein Schulmilch-Programm für arme Schulkinder, ein Brot-Subvencions- und Höchstpreis-Programm ${ }^{14}$. In den letzten Jahren, nachdem das Problembewußtsein über das Ausmaß der öffenclichen internationalen Verschuldung gewachsen ist, sind wiederholr - erwa im Falle Perus 1977 - quantitacive Obergrenzen für neue Auslandsverschuldung festgelegt worden, und es wird im Fonds derzeit diskuciert, ob diese Obergrenze in Zukunft zu den Standard-Bedingungen zählen soll. Bedenken gegen Generalisierungen dieses Mittels werden vor allen Dingen von den Vertretern entwickelter Defizit-Länder vorgetragen, die ein solches Instrumenc weiterer Disziplinierung lediglich auf Länder der Dritren Welt angewande wissen wollen.

\section{Juristische Qualifizierung der Bereitschaftskreditabkommen}

Angesichts der hervorragenden Bedeutung der Bereitschaftskreditabkommen für die Exiscenz des IWF und für nationale Wirtschaftspoliciken erscheint es ungewöhnlich, daß die IWF-Statuten sie weder in ihrer ursprünglichen. Fassung noch in der ersten großen Novellierung von 1969 auch nur erwähnen. Erst die 2. Novellierung von 1978 nimmt in Art. V eine ausdrückliche Erwähnung vor, die jedoch sehr vage bleibt und kaum die bis dahin entwickelte Verwaltungspraxis fixiert: Die Abhängigkeit der Kreditgewährung von wircschaftspolitischen Bedingungen wird nun ausdrïcklich festgelegt und dem Fonds die Kompetenz zuerkannc, derartige Bedingungen zu entwickeln (Art. V Abs. 3). Diese Formulierung bleibr ganz vage, um eine scändige, flexible "Anpassung * der Bedingungen zuzulassen's.

Durch praktische Ubung und deren - späze - Kodifizierung ist auf diese Weise ein Konflike entschieden worden, der in den Gründungsberatungen zur Errichnung des IWF eine große Rolle gespielt hate: der wesenclich von Keynes vertretenen Forderung der Schuldnerländer, bedingungslosen Zugang zu den Fonds-Mitceln zu erhaiten, war von den USA stets widersprochen worden, ohne daß eine eindeurige Statucenfestlegung erfolgt wäre. Erst die von den USA erzwungene Praxis begründete die fakcische Geltung der Bedingungen' ${ }^{16}$.

Seis ihrer ersten Formulierung im Jahre 1952 bat sich eine voliständig generalisierte Anwendung der Beistandskreditabkommen entwickelt. Bei aller graduellen Unterschiedenheit der Nebenbedingungen und trocz der an das jeweilige Schuldnerland angepaßten Restrikcionsquantitäten bleibeo die Kernbedingungen in ihrer Substanz und relativen Anwendung so gleichförmig, daß niche ohne Berechtigung von ihnen als einem weisernen Gesetz " gesprochen worden ist. Wenn Anwendung ohne Rücksicht auf konkrete Umstände des Unterworfenen, Formulierung ohne Rücksicht auf einen bereits vorliegenden Einzelfall, wenn also Abstraktheit und Alge-

If Vgl. auch die ausführlichen Schilderungen bei T. Hayter sowie bei C. Payer (FN 3 ).

1) So dic Begründung im Report by the Executive Directors to the Baard of Governars: Propased Sccond Amendmert to the Aricles of Agrecmenr, 1976, S. 20.

16 Vgl. austïhrlich J. K. Horselield, The intemational Monetary Fund r945-1965, Vol. I: Chronicle, rg6g, S. $67 f f, 267 f l, 121 \mathrm{ff}$.

17 J. Bray, Escaping from the IMF Straitjackes, in: New Statesman vom 14. 1. 1977, S. 46. 
meinheit - nichr zulerzt als Grundlage für Vorhersehbarkeit und formale Racionaljtät - zentrale Kriterien zur Bestimmung von Gesetzen sind, und selbst wenn die Anforderungen erweitert werden um sankcionsgestürzte Autorität, dann handelı es sich bei den Abkommensbedingungen um Geserze in diesem Sinne. So festgelegr sind Form und Inhal der Absichrserklärungen, daß der Chef-Jurist des IWE, ganz in der Form juristischer Gesetzeskommentienungen, den Text von Muster-Abkommen und -Erklärung Paragraph um Paragraph erläutern konnte unter der korrekten Annahme, daß die Muscer der Praxis entsprächen und mit dem für juristische Interpretation typischen Ziel, Vorhersehbarkeit und Sicherheit für (porentielle) Partner zu erhöhen.

Das Gewicht dieser Kommentierung geht sogar wejt über eine bloß̉e subjektive Interpretarion hinaus: Unter Mißachtung des auch im Völkerrecht gettenden Grundsatzes memo iudex in re suar liegt die Interpretationsherrschaft über den Inhalt der IWF-Statuten beim IWF selbst (Art. XXIX), so daß dem Chef-Juristen mit seiner Kommentierung praktisch eine ex-cathedra-Funktion zukommit ${ }^{8}$.

Obwohl substantielle Uberlegungen eine Parallelitär der Bereirschaftskredicabkommen im weiteren Sinne, d. h. inklusive seiner Bedingungen, zu den Bestimmungsmerkmalen und Zielsetzungen materieller Gesetze im freilich nationalen Bereich nahelegen, wird ihr Rechuscharakter dennoch entschieden bestritten. In seiner offiziösen Analyse des rechtlichen Charakters benenot J. Gold die wesentlichen Gründe, die gegen die Einordnung als Völkerrechısverträge sprechen sollen' 19 : Dem Partnerland solle die Demürigung erspart werden, bei Nichterfüllung bestimmter Bedingungen eines Vertragsbruchs bezichrigt werden zu müssen; es handele sich um zwei Dokumente, wobei die Bedingungen in der Absichtserklärung niedergelegt seien, die einseitig vom Partnerland erfolge und deshalb lediglich von Repräsentanten dieses Landes unterzeichnet sei; die Bezugnahme im eigentlichen Abkommen, das auch von Mitgliedern des Fonds unterzeichnet sei, stehe dem nicht entgegen, da auch diese Urkunde nicht als Vertrag qualifiziert werden könne; das Abkommen werde - im Gegensarz zu den Vertragswerken des Schwesterinstiturs, der Weltbank - nicht gemäß Art. 102 der UN-Charta registriert und veröffentlicht, wozu eine Qualifizierung als Vertrag verpflichte; es sei nicht die Praxis der Mitgliedsländer, das Abkommen nach dem je nationalen Transformationsprozeß zu ratifizieren, wie es fiur Völkerrechtsverträge zwingend vorgesehen sei; das Abkommen, besonders die Absichtserklärung, \&omme jeweils aúf Initiative der Partnerländer zuscande, auch wean der IWF "mehr oder weniger einflußreich" sei ${ }^{20}$, sie von bestimmien Maßnahmen zu überzeugen; nachdern in einer früheren Phase die Spracbe des Abkommens häufig den Charakter rechulicher Verbindlichkeit getragen habe, sei das "Ergebris des Nachdenkens beim Fonds " ${ }^{21}$ inzwischen in der formellen Entscheidung der Exekurivdirektoren vom 20. 9. 1968 niedergelegt, die sich nicht nur eindeurjg gegen rechrliche Verbindlichkeir ausspricht, sondern dekretiert: "Sprache mit dem Beigeschmack von Vertragscharakter wird in den Stand-by-Dokumenten vermieden ${ }^{22}$ - ein wabrhafr eigentümliches Dekret auf dem Hintergrund der offiziellen Doktrin, gemäß der das Abkommen auf die Iniriacive des Parnerlandes zustande kommt.

I 8 Uber die Disposizivitït des Goundsatzes wird diese weizgehende Klausel gerechufenigt, obwohl das Unterwerlungsmoment geraúe der kleineren Mitgliedsländer diesc Rechtferigung entscheidend schwächt: vgl. E. P. Hexner, Das Vcrfassurgs- und Rechlssysten des IWF, 1962, S. s 8 f; A. Broches, Intermacional Legal Aspects of ehe Operations of the World Baok, Recueil des Cours, $1959,5.301 / 3646$. 19 J. Gold, S. ${ }_{44}$ ff.

20 J. Gold, S. 48.

21 J. Gold, S. 49.

22 Exccutive Boand Decision yom 20. 9. 1968, abgedruckt in: M. Garritsen de Vries, The Internacional Monetary Fund 1966-1971, Vol. II: Documents 1976, S. 197. 
Nun stehr es Verhandlungspartnern gewiß offen, dem Ergebnis ihrer Verhandlungen rechuliche Verbindlichkeit zuzuerkennen oder nicht. Im Falle der rechtlichen Qualifizierung des Bereitschaftskreditabkommens aber gilt die vielpunkcige Argumentation zugunsten einer einseitigen Absichtserklärung keineswegs dem Ziel, das Partnerland von einer Verpflichtung freizuhalten zugunscen eines ins Belieben gestellten Verhaltens. "Im Gegenteil $\alpha$, schreibt J. Gold ${ }^{23}$ und er führt die folgende einleuchtende Begnindung an: "Es ist beschrieben worden, daß das Stand-by-Arrangement die Entscheidung ist, durch die der Fonds die Bedingungen aufstellt, unter denen Patria die Ressourcen des Fonds unter dem Stand-by-Arrangement benutzen kann und wenn Patria den Vorteil des Scand-by-Arrangements wahrnimmt, ist es auch verpflichtet, die Bedingungen zu beachten $\alpha^{24}$. Wenn aber wechseiseitige Rechre und Pflichren festliegen: das Recht des Landes, Währungen im Rahmen des Abkommens zu ziehen und das Recht des Fonds, auf Konsultacionen zu beharren, die quanticativen Durchführungs-Kriterien zu überwachen und zu messen, das Recht, bei ihrer Nichterfüllung die Kredit-Linie zu sperren, das Recht, auf Währungsrückkauf zu bestehen, dann verläßt das Arrangement den Bereich einseitiger Absichtserklärungen und efüllt gerade jene Kriterien, die gemeinhin - im nationalen Recht wie im Völkerrecht - zur Charakserisierung von Verträgen herangezogen werden. Dabei ist es nur von untergeordneter Bedeutung, ob das Arrangement in einer oder mebreren Urkunden niedergelegt wird: Das Arrangement im engeren Sinne, das die Verpflichtungen enchälc, bezieht unter ausdrücklichem Verweis die quancitariven Durchführungs-Kriterien in den VerbindlichkeitsKatalog ein, d.h. in den Rahmen jener Bedingungen, deren Nichterfüllung zur Sanktion des Kredit-Linien-Schließens und Neuverhandelns führt.

Während Verhandlungspartner festlegen können, ob das Ergebnis einer Verhandlung verbindlichen Charakter haben soll oder nicht, steht es ihnen nicht frei, eine Entscheidung über die Rechtsnatur gegen die vorliegenden inhaltlichen Kriterien zu treffen. Das ist um so weniger zulässig, als Belange nicht nur der unmitrelbar vertragsschließenden Parteien, d.h. des Fonds, des Finanzministers und/oder des Chefs der Zentralbank zur Debatte scehen, sondern weit darüber hinausgehende Interessen allgemeiner Arr, welche die Grundlage bilden exwa für Art. 102 der UN-Charta, nach denen Völkerrechrs-Verträge veröffentlicht werden müssen oder für Bestimmungen nationalen Rechts, nach denen Völkerrechtsverträge einer besonderen nationalen Behandlung, Transformation bedürfen, um innerscaatlich geirendes Recht zu werden.

Die Gründe zur Charakterisierung der Abkommen als Nicht-Vertrag vermögen denn auch gegenüber den substantiellen Erwägungen nicht zu überzeugen. Es handelt sich wesentlich um Zirkelschlüsse, bei denen von einer unerwünschten Konsequenz auf die Charakterisienung zurückverwiesen wird: die Definition wird gewähls, um die unerwünschte Konsequenz zu vermeiden. Das gilt für die bisherige Praxis, die Abkommen nicht nach Art, 102 UN-Charta zu registrieren und zu veröffentlichen ebenso wie für die bisherige Praxis der Vertragsparıner, keine nacionale Transformacion durchzuführen. Nicht diese Praxis kann über den Rechtscharakter entscheiden, sondern aus der juriscischen Qualifizierung folgt, ob die bisherige Praxis sich im Rahmen der UN-Charta bewegt. Wenn insbesondere hervorgehoben wird, daß die wirtschaftspolitischen Bedingungen in der einseitigen Absichtserklärung und nicht in einer vertraglichen Ubereinkunft niedergelegt seien, dann kornmt dieser Aussage lediglich formelle Bedeutung zu und auch dies nur in 
einem eingegrenzten Umfang: Der IWF legt den Inhalı der Absichrserklärung weitgehend fest; sie enchält die von ihm formulierten Bedingungen, unter denen er die Ressourcen-Nurzung zuläßr - in Momenten von Durchbrechung diplomatischer Ubung verweisen Angestellte des Fonds nichr ohne Stolz darauf, daß die Erklärung wesentlich von den Fonds-Missionen formuliert werden. Solcher "Enthüllung* bedarf es jedoch nicht einmal: die in jabrelanger Praxis erarbeitece regelhafte Gleichförmigkeit der in den Erklänngen niedergelegten Bedingungen, die formalen Entscheidungen der Exekutjv-Direktoren, mit deneo Form und Inhair der Bedingungen als policiesa (Art. V Abs. 3) für die Zukunft festgelegt werden, die Entsprechung von "Muster $\alpha-$ Erklärung und prakrischen Exemplaren dokumentiren über jeden Zweifel deuclich, daß nichr jeweils eine Regienung ihre wirtschaftspolitischen Absichten erklärn, sondern daß sie einen bäufig vorformulierten Vertragstext unterzeichnet, a)so substantiell eine Übereinkunft vorliegt, um die bisweilen zäh und lange verhandelt wird. Jedoch selbst formal kann die Absichtserklärnng nicht als einseitige verstanden werden, da das engere Abkommen auf die quantitariv eindeutigen Performance-Kriterien ausdrücklich Bezug nimmr. Dic formale Anweisung und Praxis des IWF endlich, möglichst jede nach Rechts-Verpflichtung klingende Sprache zu vermeiden, ändert an den inhaltlichen Charakrerisierungen nichrs. J. Gold warnt vor der Charakterisierung als Vertrag nicht zuleczt unter Hinweis auf die Erwägung, daß es für Vertragspartner erniedrigend sein müsse, des Vertragsbruchs bezichtigt zu werden. Ganz unabhängig davon, daß Nichtertüllung sich u. U. dem Einflußbereich des Partners entzieht - etwa bei weiterer Verschlechterung der terms of trade - dürfte die Erniedrigung kaum größer sein als bei der ohnehin möglichen Ankündigung, keine weireren Ziehungen zuzulassen und auf umfassenden Beratungen zu bestehen, die stets als bloße Ratschläge qualifiziert werden.

Dementsprechend erscheint es jurisrisch korrekt, der Argumentation und Praxis der Weltbank zu folgen: Die Garantie- und Darlehensvereinbarungen der Weltbank, des mit dem IWF geschaffenen Schwesterinstituss, werden wegen der in ihnen enthaltenen Zahlungs-, Konsultations- und Nebenpflicheen ohne Zögern als international-rechtliche Verträge anerkannt, nachdem die Charakterisierung von Weltbank wie IWF als Völkerrechts-Subjekt keine Schwierigkeiten mehr bereiter's', mit der selbstverständlich akzeptierten Folge der Veröffentlichung unter der UN-Charta sowie der Norwendigkeit innerstaaclich korrekter Transformation in nationales Recht. Demütigungen scheinen aus dieser juriscisch korrekten Praxis bisher nicht gefolgt zu sein, die sich ganz im Gegenteil als auch politisch weitsichtiger enweist: Die Heimlichkeit der IWF-Missionen und -Abkommen gibr immer wieder zu nbeträchtlicher politischer Unruhe Anlaß ${ }^{26}$.

Wenn dennoch die Abkommen zurecht als sneue Form von Arrangements des internationalen Rechts und Finanzwesens ${ }^{27}$ charakterisiert werden und wenn die dem Fonds seit 1978 in Arr. V Abs. 3 eingeräumce Befugnis, flexible Standards und Direktiven ("policies«) zu entwickeln, als im Völkerreche außergewöhnlicher Programmsatz zu gelten hat, dann wiederhole sich doch im Völkerrecht nur eine Entwicklung, wie wir sie im nationalen Wirtschaftsrecht bereits seit einer geraumen

25 Vgl. dic Ausführungen des Leiters der Rechtsabteilung der Weltbank, A. Broches (FN I9), S. jol ff/368 ff; ebenso Hexner, S. 11: J.-D. Kramer, Die Rechesnatur der Geschärte des Internacionaler Wahrungstonds, 1967, S. $20 \mathrm{Hf} / 10_{4} \mathrm{HF}$.

26 So etwa der Abgeosdnce des britischen Unterhauses Yates in der Debatte vom 30. I1. $\times 967$, Hansard, Sp. 647 f. Diese immer neue Unruhe führe im übrigen in Großbritannien dazu, das nach Art. 102 UN-Chana Gebotene inhaltlich nachzuvollziehen und die Abkommen zu veröffentlichen. 27 J. Gold, S. 4. 
Weile beobachcen, nämlich die Tendenz zu nicht-justiziablen Programmgesetzen, die der Exekurive weitgehendes Ermessen in ihrer Reakcion aut konjunkturelle Entwicklungen einräumen ${ }^{28}$.

\section{Kritik der Bereitschaftskreditabkommen}

Nationale politische Auseinandersetzungen um Bereitschaftskreditabkommen konzentrieren sich regelmäßig auf die folgende Aspekte: Die parlamentarische Prärogative werde ausgehöhlt und damit der Wille der Wähler verfälscht; die nacionale Selbsebestimmung und Souveränität werde untergraben; die Ubernahme von Verpflichtungen gegenüber dem IWF beinhalte stets konservative Politik und durchkreuze jeden Reformansatz. Nach der Aussage des Labour-Abgeordneten im britischen Unterhaus, M. Foot, treffen Bereitschaftskreditabkommen »nicht nur das Zentrum der Finanzstruktur, sondern darüber hinaus der Demokratic insgesamt ${ }^{29}$, während sein Kollege Mendelson die Auffassung vertrat: "Dies bedeuret, daß dem Geschäftsführenden Direktor des IWF bei politischen Entscheidungen gleicher Status eingeräumt wird wie der Regienng selbst ${ }^{\circ}$. Abbau von Sozialleistungen, Erhöhung von Mieten, Zinsen, Preisen von Konsumgütern, Telephon-, Telegraphen- und Postgebühren, Strompreisen, Verringerung von Familienunterstützungen, Erhöhung von Universitärsgebühren werden als unabweisliche inhalrliche Konsequenzen ausführlich geschildert'.

\section{Die Aushöbung der parlamentarischen Prärogative}

Die zentrale und stets zum Performance-Kriterium erhobene Bedingung aller Beistandskreditabkommen ist die Beschränkung der Ausdehnung nationalen Kredits, also eine wesentlich moneräre Maßnahme, die in den bei moderner Wirtschaftspolitik wichrigen Bereich der Programmsetzung fälk: Gesetzlich umschrieben sind nicht bescimmte Handlungsfolgen, die sich aus dem Vorliegen bestimmter Tatbestandsmerkmale ergeben, sondern Programme, welche die Regierung, die Zentralbank oder sonst eine Behörde zu angemessenem Handeln autorisieren, sobald eine - häufig nur vage umschriebene - Situation eintritt. Die Bestimmung der Geldmenge - und das IWF-Konzept der Beschränkung des inländischen Geldvolumens ist eine um gewisse internationale Aspekte erweiterte Geldmengenkonzeption " - gehört zu den zentralen Feldern solcher Programmgesetzgebung. Sie ist in die diskretionäre Beurteilung der Währungsinstiucion gestellt, d. h. entweder von einer autonomen Zentralbank - wie in der BRD oder in den USA - zu verwatten, oder von einer regierungskontrollierten Zentralbank - wie in Großbritannien: Der >Prärogative des Parlamentsı unterliegt diese Policik auch im nationalen Bereich

28 Vgl. erwa für dic Bundesrepublik: R. Knicper, Welemarks, Wirkschaftsrecht und Nationalstaat, 1976, bes. S. 25 H., S. 196 ff; für Frankreich G. Farjat, Droil Economique, 1971, 5. 389 if.

29 M. Foor, Debare vorr 5. 12. 1967. Hansard Sp. 1148; vgl. auch die Debarten im Parlamenr Neu-Seelands: vgl Hansand 3. 5. 1967, S. 92 f; 31. 10. 1967, S. 3774 f; 3. 11. 1967. S. 3970 If.

30 Mendelsan, Debalie vom 3. 12. 1967, Sp. 1174 .

31 Wyar, Debalce vom 5. 12. 1967, Hansand Sp. 1163 (Großbricannien); Kirk, Debatce vom 31, 10. 1967, S. 3774 und vom 21. 5. 1969, Hansard S. 103 (Neu-Secland).

32 Vgl. die fur die IWF-Polivik grundlegenden Arbeiten des lingjärigen Cheís der Forschungsabreilung des TWF und Besaters in volkswirtschal lichen Fragen J. J. Polak: Monetary Analysis of Income Farmation and Payments Problems, IMF Staff Papers Vol. VI No. I Nov. 1957, S. I If; Inrernational Coordinacion of Economic Policy, IMF Suff Papers, Vol. IX, No. 1. July 1962, S. 149 f. 
nicht, ebensowenig wie einer Kontrolle durch die Justiz, so daß die Klage über einen Funktionsverlust des Parlaments an der falschen Stelle ansetzt. Gerade hier nun könnte ein Grund liegen, die das zähe Festhalcen des IWF am Grundkonzept seiner Bedingungen erklärt. Es handelt sich um Bedingungen, deren nationale Anwendung ohne komplizierte parlamentarische Verfahren und ohne die Gelegenheit regierungs-oppositioneller Arrikulation möglich ist. Der diskretionärer nationaler Politik zugängliche Bereich der Geldmengebestimmung und Kreditausweirung wird durch eine formlose internationale Absprache gefült, die Regelhaftigkeit der NWFKonzeption fixiert die Regellosigkeit der nationalen politischen Situation. Das Verfahren kommt dementsprechend mit einer geringen formalen Belastung aus, kann aber ein paradoxes substantielles Ergebnis haben: Der IWF nutzr nur einen kleinen Eingriffsspielraum in nationale Wirtschaftspolitik aus; um aber in diesem kleinen Bereich zu Wirkungen zu kommen, kumulier er eine außerordentliche Kraf, die ausreicht, um weite soziale und ökonomische Bereiche zu erfassen und zu erschürern. Die Übertragung auf die anderen Bereiche ist zwar der jeweiligen nationalen Regierung überlassen, es handele sich dabei aber um Konkrerisierungen und marginale Freiheiten, die von der brennglasartigen Intensitär der Bedingungen weitgehend präformiert sind.

\section{Die Untergrabung der nationalen Selbstbestimmung}

Die Kritik am Zerfall der Souveränitär bedarf unter zwei Gesichtspunkten der Qualifizierung.

a) Der IWF verschreibc eine Wirtschartspolitik, die in den Miegliedsländern weder vollständig unbekannt noch ohne Anhänger ist. Schlichtheit und Uniformicät der Bedingungen garantieren, daß jedes antragstellende Land von vornherein eine genaue Vorstellung darüber hat, in welche Richtung die Bedingungen seine Wirtschaftspolitik bringen werden. Die regelmäßige Kombination von Beschränkung der nationalen Kredizausdehnung und Liberalisierung des Devisen- und Handelsverkehrs zu Zwecken der Zahlungsbilanz-Tarierung zieht mit Norwendigkeit bescimmte wirtschafts- und sozialpolitische Maßnahmen nach sich, die Begünstigungen und Belastungen neu verreilen und die einen gesellschaftichen Zusund mit tradicionellen Privilegienstrukturen politisch zu erhalten bestimmt sind, welche mit zunelhmender Vergesellschaftung von Produktion an ökonomiscbe Grenzen stößt. Die Bedingungen können - auch wenn sie nackt und ohne eine Reihe von Zusatzbedingungen niedergelegt sind - nicht erfülls werden, ohne staatliche Einnahmen zu erhöhen und/oder Ausgaben zu senken, eine Aufgabe, zu denen die bereits bezeichneren Instrumente zur Verfügung stehen: Erhöhung von Sceuern, Erhöhung von Gebühren sozialer Dienste, Versuche des Profitabelmachens von öffentlichen Unternehmen einerseirs, Verringerung sozialer Programme und Dienste andererseirs. Es ist ferner norwendig, die nationale Nachfrage zu d́rosseln und Exportproduktion zu unterstüızen, eine Aufgabe, die nicht ohne Einkommenskürzungen einerseits und Stimuli zugunsten der Exportindustrie - etwa in Form von Sreuemachlässen - andererseics durchgeführt werden kann. Die Verteilungseffekce solcher Programme begünstigen in der Regel gut organisierte und mächtige gesellschaftliche Gruppen, richt ohne auf den Widerstand von Benachreiligten zu stoßen.

Der Beistand des IWFF wird nun häufig in Siruationen angerufen - und das heißr nichts anderes als die Signalisierung der Bereitschaft, ein Austeritätsprogramm durchzuführen -, die gekennzeichner sind durch das Auslaulen eines Versuches, die Interessengegensätze zu harmonisieren in Reformprogrammen, welche soziale Auf- 
gaben erfüllen sollen, ohne gleichzeitig bisher Begünstigren Nachreile zuzumuren. Gerade in Gesellschaften, die in einem dramatischeren Maße die "Grenzen des Steuerstaates erreicht haben, als Schumpeter und Goldscheidt es sich bei ihrer Auseinandersetzung vor beinahe $60 \mathrm{Jahren}$ vorstellen konnten's, muß eine solche Politik zu Enttäuschungen tühren, die bei ausgebildeter Geldrvirtschaft nicht selten ihren Ausdruck in beschleunigter Inflation finden. Selbstverständlich ist die Kreditfinanzierung öffentlicher Autgaben - das deficit spending - nicht die einzige oder gar die wesentliche Quelle der Inflation, der volle Ausbau des Kreditsystems hat vielmehr seine Ursachen in der Kapitalstruktur der "privacen «nternebmen. Das Zurückdrängen der öffenclichen Aufgaben aber verfolgı das Ziel, den Verceilungskonflikt um Kredite zugunsten privater Kreditnehmer zu entscheiden. Wo der Gesamtumfang privater Nachfrage nach Krediten verringert wird, soll entsprechend selbstverständlich nicht das Kredjssystem geändert, sondern lediglich eine Schwerpunktverlagerung hin zu größerer Eigenfinanzierung gefördert werden. Da nun die Zugänglichkeit zu alternativen Finanzierungsmethoden niche von individueller unternehmerischer Geschicklichkeit allein abhängt, sondern von einer Reihe inswitutioneller Bedingungen, nämlich der Größe der Uncernehmen, ihrer Gesellschaftsform, ihrer Eingeführtheit, der Dauer von Produktions- und Verwertungsphasen, wird mir der Beschränkung der privaten Kreditexpansion ein Wertbewerbsmoment in die Volkswirtschaft eingeführt, das nicht notwendig - und damir völlig wahllos - Leistungskriterien nicht nur nicht berücksichrigen muß, sondern gar überspielen kann. Die Restriktion führt nicht zu gleichmäßig verteilter Kredirdrosselung, sondern zur vollständigen Verdrängung mancher bei vollständiger Unberührtheic anderer: wieder existieren Begünstigte und Benachreiligte, wobei die Begünstigten, d. h. diejenigen mit gutem Kredit-standing und - bei nationaler Kreditbeschränkung - mit Zugang zum internationalen Kapitalmarkt, in der Regel auch zu den politisch gut Repräsentierten gehören. Es versteht sich, daß mindestens eine Folge solcher Kreditpolicik die weitere Konzentration von Unternehmen ist.

Die Probleme verschärfen sich für Länder, in denen eine internacional konkurrenzfähige nationale Industrie erst noch aufgebaut werden soll. Wie die Geschichre der inzwischen induscriell enrwickelten Staten belegt, sind Industrialisierungsprozesse sters nur mit massiver staatlicher Unterstützung und Initiative in Gang gekommen. Dies gilt um so mehr heute, wo beginnende Industrialisienung neben den traditionellen Problemen sich dem Wettbewerb hochentwickelter, weltweit operierender Unternehmen gegenübersiehc. Staatliche Initiative kann nun durch den Aufbau öffentlicher Uncernehmen oder - in schwächerer Form - über die Unterstützung privater Unternehmen durch (Aufbau-)Kredice, Exportunterstützungen und/oder Importbeschränkungen entfaltet werden. Die Beschränkung nacionaler Kreditexpansion in Verbindung mit der Liberalisierung von Handels- und Kapicalverkehr setzr nationale Industrien unvermittelt der Weltmarktkonkurrenz aus und bedroht den Aufbau nationaler Industrien, ohne den Industrialisierungsprozeß im Weltmarkt-Kontext insgesamt zu unterbrechen. ${ }^{3+}$ Wiederum scehen den Benachteiligten - wesenclich exproprüerte Eigentümer kleinerer Produktions- und Dienstleistungseinheiten - Begünstigte gegenüber: hochentwickelte, auf dem Weltmarkt konkurrenzfähige Unternehmen, die allerdings häufig nicbc als national zu charakcerisieren sind, ferner u. U. bisher von Importbeschränkungen betroffene Importeure, Exporteure und die Eigentümer des tradjicionellen Reichtums, der stabilisiert wird.

3) Vgl. die von Hickel besorgec Neuauflage der Kontroverse Goldscheid/Schumpeter, Dic Finanzkrise dex Steuersuates - Beiträge zur politischen Okonomie der Suatsfinanzen, 1976.

$34 \mathrm{Vg}$. dic Nachweise bei Eshag/Tharg, (FN 3), Hayter (FN 3); s. auch Fröbel/Heiurichs/Kreye, Dic neue internacionale Arbeitsveilung, 1977 
Wenn Umverteilung über die staacliche Zuweisung von Geld an politische Grenzen stößt und die Grenze zu einem nicht auf individueller Verteilung abstrakten Reichturns gegründeten gesellschaftlichen Zusammenhang nicht übersprungen wird, entssehen Legitimations-Defizite gerade reformerisch auftretender Regierungen, die politische Macht kosten können. Das individuelle Interesse von Mandatsträgern kann dann mit dem Interesse der durch Reformen relativ Benachteiligten zusammentallen und den Weg zu einer Austeritärspolitik öffnen, solange die politischen Mandarsträger durch die Legitimitärskrise nicht zur Aufgabe ihrer lukrativen Posten gebracht und weil alte Begünstigungen wiederhergestellt werden. In solchen Situationen besteht erhöhte Bereitschaft, den Beistand des IWF anzurufen und das von vornherein bekannce Programm sich oktroyieren zu lassen: in den in derarcigen politischen Situacionen nicht eindeutigen Machtverteilungen vermag der IWF sein Ansehen und Gewiche in die Schale derer zu werfen, die eine ähnliche Konzeption wie er selbst verfolgen, und er vermag gleichzeitig als "Blitzableiter* (wie ein Angestellter des IWF die Funktion nannte) den Unmur neu Benachreiligter von der Regienung abzulenken, die sich als hilfloses Opfer internationalen Oktrois darzustellen vermag und nicht selten in die nationale Verdammung des harten IWF mir einstimmt.

Geschicht dies, so liegt der Souveränitätsverlust nicht darin, daß eine internationale Organisation die nationale Exekution eines nicht-national-bestimmeen Programms durchsetzt, sondern in der entschieden milderen Variante, daß in einem nationalen Konflikt der IWF Partei ergreift und auf diese Weise die Durchsetzung eines auch bereits national angebotenen Programms erleichter. Nicht der Inhalt eines Programms wird oktroyien, sondern eine nationale Front verstärkt. Dabei kann diese Front in verschiedenen Linien verlaufen: es kann sich ebenso um eine Regienung handeln, die einer starken Opposition gegenübersteht - wie erwa in Neu-Seeland 1967 - oder um eine in sich gespaltene Regierung, die Abweichler im eigenen Lager befrieden muß - wie in Großbritannien 1967 und 1969 . Daß die Kalkulation nicht ohne Risiko ist, wird durch die Tatsache belegı, daß bisweilen die BlitzableiterFunktion versagt und die Unzufriedenheit mit überspannten Austeritätsforderungen zu sozialen Unruhen führen, die dann - wie in Ghana, Sri Lanka und anderen Ländem - bevorzugt durch die eiserne Faust einer Militärdiktatur unterdrückı werden. Der IWF scheut diese Atrernarive nicht, da sie die Durchführung des Austeritätsprogramms erleichtert, wie am wiederholten Lob über die südkoreanischen und brasilianischen Verhältnisse sichrbar wird.

b) Diese Qualifizierung der Annahme, das Bereitschaftskreditabkommen höhle die narionalstaatliche Souveränitär aus, bewegt sich wesentlich im juristischen und politischen Rahmen des Souveränitätskonzepts, das allerdings auch die Debatte beherrscht. Schon diese Argumentation kommt jedoch nicht ohne einen Bezug auf den Weltmarkizusammenhang aus, d. h. eine ökonomische Kategorie. In einer weiteren Qualifizierung ist dieser Zusammenhang zu vertiefen. Das Konzept terrirorialer Souveränitä̌s' entwickelt sich nach der Zerstörung feudaler Lebenszusammenhänge in Europa als Abgrenzungs- und Konkurrenzmittel einer sich ailmählich entwickelnden Markeökonomie: Konsolidierung des Marktes nach innen, Unterstürzung der Absazzchancen heimischer Industrie nach außen, Mehrung des nationalen Wohiscandes auch aul Kosten anderer nationaler Wohlscände geben dem Begriff der cerritorialen Souveränitär die Bestimmung. Kulturelle, sprachliche, ja meraphysische Merkmale, die - wie die Begriffe der nationalen Einheit oder

3s Zum Folgenden vgl. ausfuhrlich Knieper, (FN 28); auch L. Wallerstein, The Modern World Sysern, 1974. 
Identitä - heuce den Kern des Souveränitätsbegriffs auszumachen scheinen, fehlen seinen ersten juristischen Formulierungen und hären auch nur schlecht benutzr werden können, da solche Unterscheidungsmerkmale in den ersten langen Perioden territorialer Staatlichkeit kaum existierten. Kulturmerkmale überlagerten sich und waren eher differenzierend für das Leben in Städıen oder auf dem Lande, Religionen waren gleich oder wechselcen mehrfach und gewannen erst spät eine als künstlich erkannte Bedeutung - erwa für die Konstituierung Hollands und Belgiens - selbst die Sprache war keineswegs einheitlich und gab den Territorien riche die uncerscheidende Identität. Der Begriff der nationalen Souveränität entsteht und besteht auf der Basis nacionaler Okonomie, allerdings nicht ohne im Laufe der Zeit politische und kulturelle Inhalte mir herauszubilden.

Eine mit dem Souveränitätskonzept argumentierende Position, die sich nicht im Metaphysischen der nationalen Einheit verlieren will, muß den möglicherweise geänderten Bedingungen des Weltmarktes Rechnung tragen. Der Weltmarkt aber isc $\mathrm{m}$. E. heute nicht mehr durch eindeurige nationale Okonomien gekennzeichnet, sondem durch ein hohes $\mathrm{Ma}$ an Integration und dies über klassische Handelsbeziehungen hinaus: die Internationalisierung der Produktion in multinacionalen Unternehmen hat eine Situation geschaffen, in der eindeutig ökonomische Idencifikation gerade in kleineren Nationalscascen unmöglich ist. Das rein politische Konzept der Souveränizät angesichts enger Weltmarkt-Verschränkung der Produkcion hat deshalb eine verschwindende Basis und kann kaum erfolgreich verteidigt werden, da Einmischungen wie erwa die des IWF ihren Erfolg gerade aus ökonomischer internationaler Integration und Abhängigkeir bezichen.

Dementsprecbend halte ich lediglich die Auffassung derer für konsequent, die den Begriff der Souveränirät ökonomisch und politisch füllen und deshalb zur Verteidigung der Souveränität Vorschläge machen, welche die Abwehr wirsschaftspolicischer Bedingungen zum gleichwertigen Programmpunkt mit einer (Re-)Nationalisierung der Okonomie erheben: Nationalisierung von ausländisch kontrollierten Unternehmen, Uncerbrechung der Handelsströme gehören dazu ebenso wie die Zurückweisung aller internationalen Verbindlichkeiten. ${ }^{36}$

So konsistent dieser Standpunkt erscheint, so sehr er - korrekt - mit einem umfassenden Souveränirätsbegriff operiert, so wenig läßt die bisherige Enrwicklungsgeschichte Hoffnungen auf dauerhafte, langfristige Verwirklichung zu. Wo immer bisher industrielle Produkcion Eingang in Gesellschafren gefunden hat, sei es über koloniale Uberstülpung in partiellen Sektoren, sei es über selbsigewählte Entwicklungskonzepre, hat sie die Form der Warenproduktion, der Produkrion abstrakter Werte angenommen, die prinzipiell die Verbindung zum Welcmarkt herstellt. Keine politische Deklararion über den Aufbau eines narionalen Sozialismus hat diesem Prinzip auf Dauer widerstehen können und die Denunzierung derjenigen, die das verborgen längst Wirkende an die Offentlichkeit geholt haben, als Verräter von Befreiungsbewegungen und Selbstbestimmung, hat die Hilflosigkeit schwacher ideologischer und moralischer Ricuale. Mir scheinc, daß der erste Schritc von einer Produkrionsweise unmictelbar, konkret nützlicher Gegenstände hin zur industriellen Anfertigung abstrakcer Werte eine Entwicklung einleitet, die nur noch um den Preis des Wiederherstellens der vorherigen Produktionsweise aufgehalten werden kann, die aber - einmal eingeschlagen - die Anknüpfung an den Welcmarkt prinzipiell möglich macht und langfristig nicht umgehen kann, wobei die Besonderheit gegenüber der europäischen Encwicklung zum Weltmarkc darin besteht, daß nun nicht (mehr) auf aufgeschatzten Reichtum zurückgegriffen werden

36 In diesem Sinne erwa Haycer (FN 1), Paycr (FN 3), Block (FN 8). 
kann und daß eine langsame Entwicklung der Schwierigkeit der Konfrontation mit bereits in derselben Richtung weit entwickelten Unternehmen ausgesetzt wird. Die Konfrontation erfolgt zu einem historischen Zeitpunkt, in dem die klassische Weltmarkt-Situation - nationale Unterstürzung nationalen Kapitals im Inland und Konkurrenzbeeinflussung auf dem Weltmarkr - sich verändert in Richeung auf internationale Produktion, in dem also das historische Konzept der ökonomisch fundierten Souveränität dubios zu werden beginnt und damit auch seine praktische Kraft: eine Regierung, die sich gegen Eingriffe in nationale Wirtschaftspolitik verwahrt, nicht abes das Einströmen fremden Kapitals verhindert, versucht, Symptome zu kurjeren, ohne die Ursachen zu beseitigen und - vielleicht - ohne die Ursachen dauerhaft beseitigen zu können.

Das heißr nun nicht, daß der Begriff der politischen Souveränitür und der nationaien Identität nicht wenigstens temporär genutzt werden könnte: um Abkopplungen von der Weitmarkt-Konkurrenz und den so erleichterten Aufbau einer international konkurrenzfähigen nationalen Industrie zu ermöglichen, um sonstige Konkurrenzvorteile zu erkämpfen, um (kolonialistische) Eigenrumsverteilungen zu verändern, um regionale Entwicklungsprogramme zu fördern, um insgesamt Ungleichzeirigkeiten der Enrwicklung auszugleichen mit Hilfe eines Gegengewichrs erwa gegen hoch konzentrierte, welneit operierende Unternehmen. Hier kann der Begriff der Souveränität als mächtiges Symbol wirken, als Legitimationsgnundlage und als Anreiz für snationale، Opfer. Ein solcher Gebrauch unterscheidet sich aber fundamental von der Annahme, nationale Politik sei nur etwas für nationale Pobiriker und nur solche Politik entspreche nationalen Interessen - unabhängig von der eindeutigen Existenz einer nationalen Okonomie.

Enrsprechend scheinc mir eine Ablehnung international bestimmter Wirtschaftspolioik und Geldpolitik ohne weitere Qualifizierung hilflos gegenüber dem Stand der Weltmarkrverflechtung in Form von Handel und Produktion aut der Grundlage weic entwickelter Konzentration und eines ihr entsprechenden Kredit-Geld-Systems.

\section{Der Konservativismus von Bereitschafiskedirabkommen}

Die wesentliche Kritik am Inhalt der IWF-Politik im Zusammenhang mit Bereitschaftskreditabkommen war dahin zusammengefaßt worden, daß sie grundsä̌zlich konservariv sei und - unabhängig erwa vom nationalen Wahlergebnis in Demokratien - nationale Reformpolitik international konterkariere. Der Vorwurf legt nahe, sich der sozialen Funktion der Abkommen in den Defizicländern zuzuwenden und dies unter den folgenden Aspekten: Tragen die Abkommen zur Entwicklung einer eigenständigen Industriestrukıur in diesen Ländern bei? Vermindern sie dauerhaft deren Staatsverschuldung durch Senkung des Volumens unproduktiver Staarsausgaben? Erst auf der Basis dieser Beurteilung läßt sich der Vorwurf des Konservativismus einschätzen.

Die Beistandskredicablommen haben keine konsistente Entwicklungsperspektive für Defizitländer zum Inhalt. Die Programme werden kurzfristig und erratisch eingeserzt. Auch wo sich Dauer-Kredit-Beziehungen - über Kertenprogramme von jeweils drei bis fünf Jahren - herstellen, liegt diesen keine dauerhafte EntwicklungsKonzeption zugrunde. Instrumente der konkrecen Problemdiagnose für das jeweilige Defizitland fehlen. Ungeachres der spezifischen wirtschafrlichen Siruation schreibt der bei einer fortgesetze negativen Zahlungsbilanz eines Landes um Beistand gebetene Fonds vielmehr vor, die Ausdehnung privater wie öffentlicher Kredite zu beschränken. 
Ein für anhaltend überhöht gehaltener Kreditbedarf kann neben der Verschlechterung internacionaler Handelsbeziehungen bei aller Interdependenz jedoch Unterschiedliches bedeuten: etwa das Vorliegen einer unzureichenden, unbalancierten Finanzierungsstruktur, das Unausgebildetsein eines nationalen Kapitalmarkts, anhaltende und relaziv massive Kapitalflucht, die durchschnialiche Verlängerung von Produkcionsperioden, ein Zurückfallen der Produktivicäc, das Vorliegen unzureichender Kapitalauslascang. In der Regel kumulieren mehrere der Gründe. Wenn infolge der Abkommens-Bedingungen die Zugänglichkeir zu Krediren verringerr, Steuern und Abgaben erhöht werden in Verbindung mic einer Liberalisierung des Devisenverkehrs, dann mag das Ergebnis einer solchen Wirtschafispolitik die kurzlristige Verbesserung der Zahlungsbilanz sein, substantielle, über das Kurieren isolierer Symptome hinausgehende Verbessenungen aber finden nichr nur nicht scatt, sondern sie werden im Gegensatz erschwerc: dem Aufbau eines funkrionierenden Kapicalmarktes wird mic höheren Lasten der Bevölkerung um ein weireres die Grundlage entzogen, so daß niche zuleczt deshalb die individual-wirsschaftliche Finanzstruktur sich niche verbessern kann; die Instrumente zur Verhinderung von Kapitalfluche werden gekappe und insgesamt der Aufbau eines lokalen industriellen Sektors erschwer. All diesen negativen Konsequenzen entspricht kein posiciver trade-off Gür die Encwicklung oder Produkrivität. Weder lassen sich Produktionsperioden auf diese Weise (oder überhaupt) abkürzen noch kann eine höhere Kapitalauslastung enwartet werden oder eine vermehrte Anstrengung zur Exhöhung der Produkcivicät. Gerade in Gesellschatten, in denen der induscrielle Sekcor noch nicht vollscändig ausgebildet ist, entstehen infolge der "Stabilisierungs $*$-Politik Wetrbewerbsvorteile für die Unternehmen, denen der internationale Kapicalmarkt offensteht, d. h. wesendich die multinationalen Unternehmen, die in äußerst partieller Weise zur Entwicklung beitragen und insbesondere das Beschäfrigungsproblem nicht lösen können. Da wiederum gerade in Entwicklungsländern die repatrüerten Gewinne die Kapitaleinbringung international tätiger Uncernehmen übersteigen, da ohne den Aufbau lokaler Industrie die Importabhängigkeit zum mindesten gleich bleibt, da die Exportchancen sich auf die Rohscoffe und Agrarerzeugnisse beschränken mit in der Regel exogen festgeseczten terms of trade, bleibc die Grundlage der Krise erhalcen, und auch die strikteste Austeritätspolitik vermag mittelfriscige Zahlungsbilanzungleichgewichte nicht zu verhindern.

Decaillierte Länderstudien bestätigen die plausible Folgerung, daß die Abkommensbedingungen zu Inflation und Depression, zu Unterversorgung, Arbeitslosigkeit und einer Steigerung von Bankrotten führen.

Ebenso inkonsistent ist die mit den Beiscandskreditabkommen betriebene Politik zur Reduzierung der Staatsverschuldung in den Defizitländern. Der IWF verfolgr schemarisch das Ziel einer Verbesserung der Einnahmen-Ausgaben-Relacion in den untersützten Ländern. Eine hohe Verschuldung des Staates kann aber dokumentieren, $d_{2} ß$ hohe Ausgaben für Ausbau und Erhalt einer Infrastruktur, für repressive Apparare als Mittel der 'Befriedung nach innen, für militärische Apparace als Mittel der Außenpolitik, für den Ausbau bzw. den Erhale der industriellen Produkrion, für die Finanzierung sozialer Leistungen getätigt werden, denen keine entsprechenden Einnahmen in Form von Steuern gegenüberstehen, sei es, weil das Steversystem nicht voll entwickelt ist, sei es, weil eine politische, gesellschaftliche Grenze steuerlicher Belastbarkeit erreicht ist. Die Ausgaben mögen durch konjunkturelle Erwägungen mir begründer sein, etwa im Vollzug antizyklischer Fiskalpolitik. Jedoch selbst in Ländern mit relariv hochentwickelter Berücksichcigung von Konjunkturerwägungen bei der Haushalısgestaltung machı der dieser Berücksichrigung entsprechende Anteil der öffendichen Ausgaben jeweils nur einen Bruchteil aus; er 
kann deshalb im weiteren vernachlässigt werden. Der ganz überwiegende Teil setze sich aus Ausgaben zusammen, den die politisch verantwordichen Budget-Gestalter außerhalb kurzfristig konjunkrureller Erwägungen für notwendig halcen. Die Tatsache, daß ein beträchtlicher Anteil des Haushalts über Kredite und nicht über Einnahmen des Staates finanzier wird, deutet dabei darauf hin, daß die politisch Verantwortlichen die Einnahmequellen des Staates für ausgeschöpft halten, wobei die Eirschätzung dieses Zustandes aus wahltakcischen Gesichrspunkten (mic-)begründer sein kann.

Wenn der IWF mic seinen Kredicbedingungen schemacisch die Verschiebung einer vorgefundenen Einnahme-Ausgabe-Relation durchzuseczen versucht, also etwa eine Erhöhung der Einnahmen, eine Verringerung der Ausgaben oder - wie in der Regel - eine Kombinacion beider, dann tur er das aut der Grundlage einer Einschärzung der nationalen ökonomischen, politischen, sozialen Lage, die von der Einschärzung der nationalen Polisiker abweiche.

Es wäre nun immerhin denkbar, daß der IWF mit seiner von nationalen Politikern abweichenden Einschärzung norwendiger Ausgaben und erzielbarer Einnabmen in diesem Land eine ,Lücker überbrückc, die vielen Defizíländern eigen ist: daß nämlich ökonomische und politische Herrschaftsstrukturen zu eng miteinander verschränkt sind, um im nationalen Rahmen auch nur gesamtkapitalistisches Algemeininceresse zu realisieren. Angesichrs dieser Situation könnte dem Eingreifen des IWF die Rolle zukommen, dem im bürgerlichen Staat verkörperten besonderen Allgemeininteresse zum Durchbruch zu verhelfen.

Um diese Annahme auf ihre Realitäc überprüfbar zu machen, ist eine theoretische Vorklänng erforderlich: In bürgerbichen Gesellschaften verdankt sich die Existenz des Srates - und das beißr insbesondere seine in Einnahmen und Ausgaben materialisierte Existenz - nicht dem voluntaristischen Belieben von Politikern und Bürokraten, die in der immer weiteren Ausdehnung der Staatssphäre ihre Machtgelüste befriedigen. Vielmehr ist der Staat die der in Konkurrenz erfolgenden Produttion abstrakten Reichrums encsprechende Inscanz, der es im wesentlichen obliegt, die Konkurrenz in einem den bürgerlichen Gesellschaften eigentümlichen Allgemeininteresse aufzuheben. Dabei ist weder die inhaldliche Definition des Norwendigen ein für alle Mal festgelegt, noch garanciert, daß das Norwendige sich (automatisch) durchsetzen wird, noch daß es sich überhaupt durchsetzen wird, noch daß jede durchgeführte Maßnahme in kurztristiger Analyse sich als Norwendiges bestimmen läßt. Die Veränderung der Produktionsbedingungen läßc neue Manilestationen des Allgemeininteresses, neue Defizite an unmittelbarer Gesellschafdichkeit entstehen und damit neue Aufgaben des Staates. Die relative Autonomie des Politischen, d. h. auch des praktischen Personals, macht es, ebenso wie die Anarchie der Marktökonomie, wahrscheinlich, daß das der Produkcion entsprechende Allgemeininteresse sich nicht zügig, nach bescer theorecischer Einsicht, durchsetzt, sondern zögernd, auf Umwegen über die Befriedigung von Partikularinteressen, häufig erst über die Erfahrung katastrophaler Fehlentwicklungen. Da die Erfüllung von Aufgaben mit Kosten verbunden ist, die in den individuell verteilungstähigen Mehrwert schneiden, und da Poliviker in der Regel keine unmictelbaren Vorreile von ibrer Erfüllung haben, ist es eher wahrscheinlich, daß diese hinausgezögert, zurückgenommen, unvoilszändig und unzureichend angegangen als daß sie übererledigr werden. Ein solches der bürgerlichen Gesellschaft eigennümliches Allgemeininteresse als von dort ebenfalls bestehenden Parrikularinteressen unterschieden ist Realität. Die Existenz von Klassen und Konkurrenz widerspriche dem niche, sondern ist seine fundamentale Bedingung: die bürgerliche Gesellschaft müßte zusammenbrechen (und dies richt, um unter seinen Trümmern die Fundamente des Sozialismus 
freizulegen), wenn Klassen und Konkurrenten sich ausschließlich in Verfolgung ihrer Individual-Interessen aufeinander bezögen, und es keine Instanz gäbe, die in (historisch) bestimmren Bereichen das Interesse aller, auf der Oberfläche catsächlich prinzipiell Gleicher und Freier verträte.

Das Festhalten an der Exisrenz von Algemeininteresse und seine Unterscheidung von Partikularinteressen hat für die Einschätzung der Bereitschaftskreditabkommen Bedeutung. Die besondere Form der politischen Organisacion in bürgerlichen Gesellschaften - und das gilt für Diktaturen und (wenn auch in geringerem Maße) für parlamentarische Demokratien - macht es möglich und wahrscheinlich, daß die politischen Akteure das Allgemeininteresse nur (wenn überhaupt) über den Umweg ihres partikularen Interesses vertreten. Da die unmittelbare gesellschaftliche Artikulation und Durchsetzung von gesellschaftlichen Bedürfrissen in kollektiven Prozessen gerade der oberflächlichen Individualicät widersprichc, der der (bürgerliche) Staac seine Existenz verdankr, handels es sich dabei um ein unauflösliches Problem, das noch verschärft zu werden scheint, wo eine territoriale politische Einheit sich - häufig im Gefolge kolonialer Herrschaft - neu bilder über einer Okonomie, die noch weitgehend durch ein materielles und formeiles Zusammenfallen ökonomischer und politischer Macht gekennzeichnet ist, und wo die plörzlich eingeserzte bürgerliche politische Struktur den tradicionellen Zusammenhang dadurch wiederherstellc, daß sie die ökonomische Macht mitzuerobern sucht.

Wird nun der Umweg über das Partikularinteresse, der im übrigen kein Privileg schwacher Defizicländer ist, durch den Einfluß des IWF abgekürzt, der selbsı keine Partikularinteressen in den Ländern verfolgt? Tilgen seine Scabilitätsprogramme Staatsausgaben um den Anceil persönlicher Bereicherung, unprodukriven Pomp. leistungswidrige Bestechungen, überhöhte Militärausgaben oder zwingen sie sie doch in kJeinere Dimensionen und lenken so Reserven auf die Erfüllung von Allgemeininteressen?

In den mir vorliegenden Dokumenten lassen sich derartige Bedingungen nicht entdecken, weder offen ausgewiesen noch - um Peinlichkeir zu ersparen - in versteckrer Form. Ob in den vertraulichen Verhandlungen auf sie hingewirkt wird, läßt sich nicht sagen. Der Augenschein spricht eher dagegen: übermäßiger Pomp, das Bekanntwerden größerer Bestechungsskandale oder die Existenz kostenintensiver Polizei- und Milieärapparate auch in verhältnismäßig kleinen Ländern stellen offensichtlich keinen Hinderungsgrund für guce Beziehungen zum IWF dar. Eher scheint das Gegenteil der Fall zu sein, wie sich an den dauerhaft günsrigen Beurteilungen Süd-Koreas, der Phillipinen und Brasiliens zeigt, alles Länder, in denen aufwendige Polizei- und Militärapparate, Korruption und Regierungspomp nebeneinander in auffälligem Maßse existieren.

$\mathrm{Da}$ aber im Gefolge von Kredit-Bedingungen Polizei- und Militärapparate nicht kostensenkend verringert, sondern eher kostentincensiv verstärkt werden, hat seine Logik in den Austeritärskonzepten. Sie lassen sich kaum ohne schwere soziale Spannungen durchführen, welche staatliche Repression auf der Skala von intensiverer Streikbekàmpfung bis hin zur offenen Milizärdiktacur zur regelmäßigen Folge haben. Nur in den allerselzensten Fällen gelingt es der Regierung, die Masse der Bevölkerung davon zu überzeugen, warum sie die wesenclichen, harten Folgen der Krise cragen soll, die doch nicht durch Faulheic und Arbeitsunwilligkeit entstanden ist. Die Folge ist, daß Staatsausgaben im Anschluß an Beistands-Kreditabkommen nich gleichmäßig gesenkt werden, sondern daß eine Umlenkung vonstatten geht. Repressive Auf- und Ausgaben des Siaates werden zum mindesten relaciv verstärkc, während andere Auf- und Ausgaben unverhälmismäßige Kürzungen erfahren.

Wenn politische Gründe ein Zurückdrängen repressiver Funktionen des Staats 
verhindern - Gründe, die empirisch gut belegt sind -, bleibr die nacionale Exekution der Abkommensbedingungen auf die Kürzungen von Sozialleistungen (die sich über Gebührenerhöhung oder Leisungsbeschneidung erreichen läß $(\mathfrak{t})$, auf die Verminderung des Ausbaues allgemeiner Produkrionsbedingungen und auf die Durchserzung (oder Erhöhung) der Profitabilität öffentlicher Unternehmen verwiesen. Die stärkere Eröffnung neuer Quellen zur Finanzierung der öffentlichen Aufgaben - erhöhre Steuereinnahmen und die Inanspruchnahme des privaten Kapitalmarktes - ergänzen die abkommens-induzierzen Veränderungen.

Sowenig also der IWF mit seinen Beistandskreditabkommen zur Entwicklung einer daverhaften Industriestruktur in den Defizitländern beiträgt, sowenig trägt er zur Stabilisierung von bürgerlicher Sraaclichkeit als Instrument des oberflächlichen gesellschaftlichen Zusammenhalt partikularer und antagoniscischer Interessen bei. Eher verrieft er die ökonomischen und sozialen Problemlagen und verschärft die Konfliktpotentiale, anstatt sie abzumildern.

Wie läßt sich eine solche Politik gesellschaftspolitisch qualifizieren? ,Progressive in einern emphatischen Sinne ist sie gewiß nicht. Ist sie ,konservative, wie Kritiker behaupten? Wenn der Begriff des Konservativen einen präzisen Sinn behalten und nicht einfach zum diffusen Schlagwort abgenutzt werden soll, dann ist auf zwei ihm innewohnenden Elementen zu insistieren: Konservarive Politik zielt darauf, den herrschenden gesellschaftlichen Zustand - also etwa Privareigentum und Individualismus - politisch zu bewahren, und das heißh, Gefährdungen dieses Zustandes infolge ökonomischer, sozialer oder politischer Veränderungen und Ungleichgewichtslagen durch ausgleichende, inregrierende, scabilisierende Gegenmaßnahmen entgegenzutreten, den Versuch also zu unternehmen, die entstandenen Konfliktpotentiale durch neve Gleichgewichtslagen zu eliminieren. Einen solchen Begriff des Konservariven hat etwa J. M. Keynes verfolgt, wenn er in der Absicht, ein nSystem des Staatssozialismusu zu verhindern, das ngemäßigt konservarive« Programm für eine "ziernlich umfassende Verscaatichung der Investition ... als das einzige Mitrel zur Erreichung einer Annäherung an Vollbeschäfrigung a formulierre ${ }^{37}$.

Im so präzisierten Sinne isc konservarive von reakcionärer Politik abzugrenzen. Diese suche den gesellschaftlichen Zustand zu erhalten, ohne auf eingerretene Konfliktlagen und -potentiale mit dem Versuch der integrierenden Schaffung neuer Gleichgewichte zu antworten. Die reakrionäre Erhalung des status quo bleibr letztlich nur noch durch Repression möglich - Repression, die allerdings Konfliktagen eher noch zuspirzt als bewäligr, die folglich rrorz aller Zufügung individuellen Leids langfristig erfolglos bleibr. Dafür legr die Encwicklung in einigen der vom TWF sgeförderten Länder beredres Zeugnis ab. In diesem Sinne ist denn auch die IWF-Politik nicht angemessen als sonservativ zu charakterisieren. Sie ist reaktionär und irracional gerade auch dann, wenn sie am Maßstab der Racionalizär der bürgerlichen Gesellschaft gemessen wird. Gleichzeicig ist sie erfolglos, wobei die Kriterien für Erfolg aus Arc. I der IWF-Stacuten zu encnehmen sind: Es fehlt bisher jeder Hinweis, daß Bereirschaftskreditabkommen die incernationale Währungsintegration, das gleichgewichtige Wachsen des Welthandels, ein hohes Beschäfrigungsniveau, hohe Realeinkommen sowie die produkciven Ressourcen aller Mitgliedsländer fördern.

37 J. M. Keynes (FN 7). S. 3181. 
Austerität und Militärdiktatur mögen in einem engen Bedingungsverhältnis zueinander stehen. Beide scheinen extreme polirische Manifestationen eines ökonomischen Systems zu sein, in dem die Produktion nichı unmirtelbar auf die Befriedigung von Bedürfnissen der produzierenden Menschen zielt, sondern in dem abstrakte Werte produzierc und die Bedürfnisbefriedigung nur über den Tausch dieser abstrakcen Werte ermöglicht wird. Die Trennung von Bedürfnisbefriedigung und Produktion verlangt nach Menschen, die ihre Arbeitskraft einsetzen, obwohl die Ergebnisse des Arbeitsprozesses ihrer Bedürfnisbefriedigung nicht unmitzelbar dienen, wobei der Einsacz selbst nichı dem Prinzip der Lustgewinnung folgt, sondern den dem Luscprinzip gegenüber gleichgültigen Erfordernissen der Produktion abstrakter Werte. Die solcher Produkcion angemessenen Eigenschaften entsprechen offensichtlich weder einer narürlichen menschlichen Anlage noch sind sie jeder hiscorischen Produksionsform adäquat. Wie die europäische Sozialgeschichte reichlich belegt, bedurfte es langer und bisweilen außerordentlich gewaltsamer Disziplinierung, um die Fabrikrugenden in die Geister und Körper der Produzenten abstrakter Werte einzuhämmern, und selbst dies ist nur oberflächlich gelungen, wie nich nur das Material psychischer Erkrankungen zeigt, sondern auch die Leichtherzigkeit, mit der der Produkcionsdisziplin zu entrinnen versucht wird, sowie endlich die fortwährende Disziplinierung, die im Produkrionsprozeß ständig aufrechı exhalten werden muß. Wo schon Länder mit langer Tradition der Warenproduktion auf immer emeute kollektive wie individuelle Zurichtung nicht verzichten können, sind Gesellschatten, die sich auf der Bahn der industriellen Entwicklung befinden, um so mehr auf die Zurichtung und Entwicklung von Fabriktugenden angewiesen, je frischer die Erinnerung und sogar die parcielle Exiscenz einer andersarrigen Produktionsstruktur und Lebensweise ist.

Der Begriff der Entwicklung atmet den Fortschrittsglauben der bürgerlichen Gesellschaft - er impliziert das Erreichen einer höheren Stufe. Wie stark dieser Inhal das Denken beherrscht, zeigen Stellungnahmen sbürgerlicher * Autoren, die den Prozeß der Industrialisierung in einem Land loben und als bitteren Tropfen, als düsteren Fleck des heiseren Bildes die politische Unterdrückung kritisieren und aus ehriichem Herzen die Beseitigung dieses negativen Moments anregen, aber auch "sozialistischer* Autoren, die das Elend und die brurale Disziplinierung der Massen erschreckt und die angesichts dieser Erfahrung das Vorhandensein von Enrwicklung ganz und gar ableugnen. Wie Peter Evans in einer Fallstudie ${ }^{8}$ über Brasilien nachgewiesen hat, findet Encwicklung, Industrialisierung, Anschluß an die bereits entwickelten Länder tatsächlich starc. Resigniert aber erkennt er, daß die Entwicklung nicht zu einem höheren Grad an Humanisierung führt - eine Feststellung, die niemanden überrascht, der den Zustand der hochentwickelten Industrie-Gesellschaften analysiert.

Wie bereics an anderer Stelle ausgeführt, isc mir auch keine policische EntwicklungsAlternative geläufig, die die Disziplinierung uncer die Erfordernisse der Warenprodukcion vermeiden könnte, insbesondere durch Vermeidung der Warenprodukcion selbst. Diese erscheint mir ganz unentrinnbar zu sein, wenn nur erst die Encwicklung zur Industrialisierung eingeschlagen ist. Disziplinierung ist der norwendige Preis dieser Entwicklung. Das heißt natürlich nicht, daß jede Entwicklung gleichförmig und gleichförmig gewaltsam verlaufen müßre. Politische Unrerschiede kön-

38 P. Evans, Multinationals, State-owned Corporations and the Transformation of Imperialism: A Brazilian Case-Siudy, in: Economic Development and Cultural Change, Vol 26 No. 1. Oct. 1977. S. 43. 
nen eine gewalrige Differenz für das Ausmaß an Leiden für die der Disziplinienung Unterworfenen eröfinen - am Prinzip norwendiger Disziplinienung jedoch scheint sich nichts zu ändern, wobei die Erfahrung zeigt, daß deren Charakterisienung als »sozialistische oder "kapiralistische « Disziplin für die Betroffenen keinen nennenswerten Trost bereithält.

Nun läßs sich der TWF sicherlich nichı für das Vordringen der Warenproduktion verantwortlich machen, und seine Charakterisierung als Wegbereiter des Kapizalismus erscheint mir unkorrekt. Die Verantwortlichkeit ist beschränkter, aber immer noch gro $B$ genug. Sie besteht darin, $d_{a} B$ die verordneten und reilweise nur zu willig aufgenommenen Austerisärsprogramme eine Entwicklungsvariante aufdrängen, in der Disziplinierung unter besonders inbumanen und unsozialen Bedingungen vonstacten geht. 